\title{
Perfil hematológico del guajolote criollo de traspatio en la región de Tecamachalco, Puebla.
}

Hematological profile of the backyard creole turkey in the region of Tecamachalco, Puebla.

Reséndiz Martínez Roberto $^{1 凶}$, Salvador Romero Castañón ${ }^{1}$, Herminio I. Jiménez Cortez ${ }^{1}$, Briseida Lucía Castro Bautista ${ }^{1}$.

${ }^{1}$ Facultad de Medicina Veterinaria y Zootecnia, Benemérita Universidad Autónoma de Puebla. Domicilio conocido, El Salado, Tecamachalco, Puebla, México; vetuap@yahoo.com.mx. Teléfono y fax: 012494220178.

${ }^{\square}$ Autor para correspondencia

Recibido: $15 / 07 / 2017$

Aceptado: 10/12/2017

\section{RESUMEN}

Los guajolotes criollos en el medio rural han tenido un papel de suma importancia ya que han aportado a la población humana proteínas de alta calidad biológica (carne y huevo) por lo general en condiciones adversas. La presente investigación tiene como objetivo describir el perfil hematológico del guajolote criollo de traspatio de la región de Tecamachalco, Puebla. Se seleccionaron y adquirieron 23 guajolotes criollos (GC), que se mantuvieron en confinamiento durante un mes alimentándose con maíz molido y alfalfa, similar al lugar de origen. Para el estudio hematológico se tomó una muestra de dos ml de sangre del ala. Se colocó en un tubo vial con anticoagulante EDTA rotulándose para su identificación. Se realizaron las siguientes determinaciones: Hematocrito (método del Micro hematocrito con tubos capilares, centrifugados a 12.000 r.p.m. durante 5 minutos), Hemoglobina (método de Drabkin), Glóbulos blancos (Neubauer) y Formula leucocitaria (recuento diferencial en frotis según Giemsa). Los resultados obtenidos fueron los siguientes: para hematocrito, proteínas plasmáticas, leucocitos totales, linfocitos, heterófilos, eosinòfilos, basófilos y monocitos, 49.39\%, $5.56 \mathrm{~g} / \mathrm{dl}, 22.55 \mathrm{x} 10^{3} / \mathrm{ul}, 50.48 \%$, $35.35 \%, 11.57 \%, 1.65 \%$ y $5.87 \%$, respectivamente. Los valores de referencia son: $49.39 \%, 5.56 \mathrm{~g} / \mathrm{dl}, 20$ x $10 \%$ ul, $50 \%, 43 \%, 19 \%, 3 \%$ y $9 \%$, por lo que se considera mínima la diferencia entre lo obtenido y los rangos referenciales. Esta información hematológica de los GCM en las condiciones descritas es la base para futuras investigaciones sobre esta especie animal, en particular para los estudios de resistencia a enfermedades y otros factores ambientales.

Palabras clave: Hematologia, Guajolote, Heterofilos, Leucocitos, Proteínas plasmáticas.

\begin{abstract}
ABSTRAC
The creoles turkeys in rural environment have played an important role as they have provided the human population with high biological quality proteins (meat and eggs) usually in adverse conditions. This research aims to describe the haematological profile of the creoles turkeys of the Tecamachalco region, Puebla, Mexico. 23 creoles turkeys (GC) were selected and acquired, which were kept in confinement for a month, feeding on ground corn and alfalfa, similar to the place of origin. For the hematologic study,

Revista Científica Biológico Agropecuaria Tuxpan 5 (2) ISSN: 2007-6940
\end{abstract}


a sample of two $\mathrm{ml}$ of the wing blood was taken. It was placed in a vial with anticoagulant EDTA. The following determinations were made: Hematocrit (Micro Hematocrit method with capillary tubes, centrifuged at 12,000 r.p.m. for 5 minutes), hemoglobin (method of Drabkin), and white blood cells (Neubauer) and Formula leukocyte (count differential smeared according to GIEMSA). The results obtained were the following: for hematocrit, plasma proteins, total leukocytes, lymphocytes, heterophiles, eosinophils, basophils and monocytes, 49.39\%, 5.56 g/DL, 27.89 x 103/ul, 50.48\%, $35.35 \%, 11.57 \%, 1.65 \%$ and $5.87 \%$, respectively. The reference values are: $49.39 \%, 5.56 \mathrm{~g} / \mathrm{DL}, 20 \mathrm{x}$ $103 / \mathrm{UL}, 50 \%, 43 \%, 19 \%, 3 \%$ and $9 \%$, so it is considered minimum the difference between the obtained and the reference ranges. This hematologic information of the GCM under the conditions described is the basis for future research on this animal species, in particular for studies of resistance to diseases and other environmental factors.

Keywords: Hematology, Turkey, Heterophile, Leukocytes, Plasma proteins.

\section{INTRODUCCIÓN}

Entre los animales que se crían a traspatio encontramos a las gallinas, guajolotes, cerdos, conejos y patos. Ambos brindan alimentos variados a la dieta familiar, además de ingresos económicos, con la venta de excedentes que se producen, o bien son formas de ahorro para cualquier eventualidad que se presente en la familia. En principio es importante que los animales tengan un lugar específico para su mejor manejo, con ello se evitan enfermedades y se logra una mejor alimentación. Los guajolotes criollos en el medio rural han tenido un papel de suma importancia ya que han aportado a la población humana proteínas de alta calidad biológica (carne y huevo) por lo general en condiciones adversas (López, 1997).

La hematología es una de las herramientas de diagnóstico más útiles, y al mismo tiempo más infrautilizadas de la práctica veterinaria. La composición de la sangre cambia rápidamente en respuesta a la enfermedad. El hemograma completo proporciona una excelente información sobre el estado de salud de un paciente. Los hemogramas aportan datos cuantitativos (recuentos totales celulares, recuentos diferenciales totales, índices eritrocíticos, etc.) y cualitativos (morfología celular en la extensión sanguínea). Los estudios de hematología y química sérica pueden ser herramientas sumamente útiles con una variedad de aplicaciones. La obtención de muestras de sangre constituye una forma no destructiva de obtener grandes cantidades de información que puede ser de utilidad para tomar decisiones referentes al manejo y conservación de una especie determinada. (Franzmann et al., 1978; Lochmiller et al., 1984).

Asociado a lo anterior la realización de estudios de hematología y química sérica en aves tiene especial importancia, ya que la observación de signos clínicos de enfermedad por lo general es enmascarada hasta sus etapas tardías, lo que es principalmente evidente y una necesidad en especies susceptibles a predación (Rosskopf et al., 1991; West et al., 2002). Desde el punto de vista clínico el establecimiento de valores de referencia para hematología y química sérica aviar permite incorporar datos clinicopatológicos a la anamnesis y el examen físico de un paciente. Esto ayuda al clínico a lograr una mayor comprensión de las organopatías y los cambios bioquímicos y fisiológicos que estas puedan causar y por lo tanto mejorar el manejo de estas especies (Peinado et al., 1992; Tell et al., 1992; Hochleithner, 1994; García et al., 2002). 


\section{MATERIALES Y MÉTODOS}

La investigación se realizó en el Municipio de Tecamachalco, Puebla, Mex. Se localiza al sureste del Estado de Puebla, a 56.7 kilómetros de la capital de la entidad, se ubica en los paralelos $18^{\circ} 52^{\prime}$ '57" latitud norte y a $97^{0} 43^{\prime} 49^{\prime \prime}$ latitud oeste. Para el estudio se seleccionaron 23 GCM clínicamente sanos los cuales se mantuvieron en confinamiento durante un mes alimentándose los animales con maíz molido y alfalfa, similar al lugar de origen. Para el estudio hematológico se tomó una muestra de dos $\mathrm{ml}$ de sangre a partir de la vena cutánea cubital a nivel de la articulación del codo del ala. Se colocó en un tubo vial con anticoagulante EDTA y se realizaron las siguientes determinaciones: Hematocrito (método del Micro hematocrito con tubos capilares, centrifugados a 12.000 r.p.m. durante 5 minutos), determinación de proteínas plasmáticas (método de Biuret), Glóbulos blancos (Neubauer) y Formula leucocitaria (recuento diferencial en frotis según Giemsa) (Nietfeld et al., 1994). Se verificaron si los datos obtenidos seguían una distribución normal (método de Kolmogorov - Smirnov) y si cumplen con la homogeneidad de varianza (Prueba de Levene). Al no presentarse una distribución normal, se realizó la prueba no paramétrica de Kruskal - Wallis para la comparación de la variabilidad biológica de medias (guajolote de traspatio sobre los valores de rango), con el apoyo del paquete estadístico IBM SPSS 15 para Windows, a un nivel de significancia $P \leq 0.05$

\section{RESULTADOS Y DISCUSIÓN}

Los resultados obtenidos fueron los siguientes: para hematocrito, proteinas plasmaticas, leucocitos totales, heterofilos, eosinofilos, basofilos y monocito; 49,39\%, $5.56 \mathrm{~g} / \mathrm{dl}$, $50.48 \%, 35.35 \%, 11.57 \%, 1.65 \%$ y $5.87 \%$ (tabla 1 y grafica 1$)$.

Los valores de referencia son: $49.39 \%, 5.56 \mathrm{~g} / \mathrm{dl}$, $20 \times 103 / \mathrm{ul}, 50 \%, 43 \%, 19 \%, 3 \%$ y $9 \%$, por lo que se considera mínima la diferencia entre lo obtenido y los rangos referenciales (Dein et al., 1982; Maxwell et al., 1993) (tabla 2).

El desarrollo de valores de referencia para hematología y química sérica obtenidos a partir de poblaciones de guajolotes sanos es importante debido a que estos proveen la línea base e información diagnóstica que permite evaluar el estado de salud o enfermedad de poblaciones silvestres capturadas por metodologías similares (Borjesson et al., 2000). Los pollos y pavos domésticos presentan por lo general mayor abundancia de linfocitos en el leucograma (Borjesson et al., 2000., Drew, 2003) patrón que fue observado también en los guajolotes de traspatio de estudio. La tabla no. 3 muestra la media e intervalo de confianza del 95\% y rango mínimo y máximo de los 8 parámetros hematológicos determinados en los 23 Guajolotes incluidos en el estudio.

En el estudio realizado por Girish y col. (2005), las concentraciones de hemoglobina, hematocrito y concentración corpuscular media de la hemoglobina (MCHC) no fueron afectados significativamente por la dieta. La alimentación de granos contaminados se redujo $(\mathrm{P}<0.05)$ recuentos totales de linfocitos a la semana 3 . Sin embargo, no hubo cambio en el total de glóbulos blancos y otros recuentos diferenciales de leucocitos. Hubo un aumento significativo ( $\mathrm{P}$ $<0.05$ ) en el porcentaje de poblaciones de linfocitos CD4 + durante la semana 6 en aves alimentadas con granos contaminados. Sin embargo no hubo cambio, en el porcentaje de poblaciones de linfocitos B y CD8+ (Girish et al., 2005). En el presente estudio no se utilizó alimento contaminado observándose coincidencias en los parámetros hematológicos, 
en donde no fueron afectados significativamente por la dieta. Los estudios presentados por Lashev et al., 2015, no presentaron diferencias interespecies estadísticamente significativas o diferencias relacionadas con el sexo en los recuentos de leucocitos. También se registraron tendencias para mayores relaciones heterofilos/linfocitos (H/L) en aves machos. Las diferencias relacionadas con la raza, resultado de varios grados de domesticación, no fueron identificadas. Los resultados en dicho análisis fueron obtenidos por diferentes investigadores, en diferentes laboratorios, latitudes y condiciones climáticas. De tal forma que los valores hematológicos y referentes a las células blancas, coinciden con la presente investigación. Los resultados hematológicos mostraron que no hay densidad de población como causa de diferencia significativa de hematocrito, recuento de glóbulos rojos, hemoglobina, volumen corpuscular medio (MCV), cantidad de hemoglobina corpuscular media $(\mathrm{MCH})$, concentración de hemoglobina corpuscular media (MCHC), recuento de trombocitos, total recuento de leucocitos y leucocitos diferenciales. Tampoco se pudo determinar ninguna diferencia significativa mediante el cálculo de la relación Heterofilos/Linfocitos, que se usa como indicador de estrés (Hafez et al., 2016). Los resultados del estudio hematológico del guajolote criollo obtenidos en la presente investigación presentan relación con los obtenidos por Hafez. Cetin (et al, 2005) observó que la suplementación probiótica causó aumentos estadísticamente significativos
$(\mathrm{P}<0.05)$ en el conteo de eritrocitos, concentración de hemoglobina y valores de hematocrito, pero la suplementación con mannanoligosaccharide (MOS) no tuvo un efecto significativo $(\mathrm{P}>0.05)$ en estos parámetros. Los recuentos totales de leucocitos leucocitarios y diferenciales no se vieron afectados por la suplementación con MOS y probióticos en la dieta. En la presente investigacion de guajolotes criollos no se utilizó suplementación alguna, de tal forma que no se presentaron aumentos estadísticamente significativos en los en los valores hematológicos. Los resultados obtenidos por Katarzyna et al., 2015, mostraron que los factores de estrés aplicados causaron una disminución significativa $(\mathrm{P} \leq 0.05)$ en el nivel de lisozima y $\%$ de células fagocitìcas, así como una reducción no significativa en la actividad metabólica de los heterofilos. Bajo la influencia del estrés se observó un aumento $(\mathrm{P} \leq 0.05)$ en el porcentaje de monocitos y basófilos. El uso de la preparación de aloe mejoró algunos indicadores de inmunidad (aumento, $\mathrm{P} \leq 0.05$, lisozima y $\%$ de células fagocitícas) en las gallinas de pavo. Para aliviar el efecto negativo del estrés sobre las reacciones inmunes, consideraron la administración de suplementos con extracto de aloe suplementado con resveratrol y vitamina $C$. Existe diferencia entre los resultados obtenidos en dicha investigación, ya que no hubo aplicación del estrés y no se utilizó ninguna preparación, por lo que se considera mínima la diferencia entre lo obtenido y los rangos referenciales.

TABLA 1.- Valores hematológicos obtenidos del guajolote criollo.

\begin{tabular}{|l|l|l|l|l|l|l|l|l|}
\hline $\begin{array}{l}\text { Clave del } \\
\text { Guajolote }\end{array}$ & Hematocrito\% & $\begin{array}{l}\text { Proténas } \\
\text { plasmáticas g/dl }\end{array}$ & $\begin{array}{l}\text { Leucocitos } \\
\text { totales x10 }\end{array}$ & $\begin{array}{l}\text { Linfocitos } \\
\%\end{array}$ & $\begin{array}{l}\text { Heterófilos } \\
\%\end{array}$ & $\begin{array}{l}\text { Eosinófilos } \\
\%\end{array}$ & $\begin{array}{l}\text { Basófilos } \\
\%\end{array}$ & $\begin{array}{l}\text { Monocito } \\
\%\end{array}$ \\
\hline 1 & 30 & 5.5 & 20.8 & 41 & 10 & 41 & 4 & 4 \\
\hline 2 & 60 & 4.0 & 23.2 & 33 & 38 & 22 & 1 & 8 \\
\hline 3 & 50 & 5.5 & 23.6 & 79 & 11 & 4 & 1 & 5 \\
\hline
\end{tabular}


Reséndiz et al., 2017

\begin{tabular}{|l|l|l|l|l|l|l|l|l|}
\hline 4 & 50 & 5.5 & 7.8 & 95 & 80 & 14 & 1 & 10 \\
\hline 5 & 65 & 6.8 & 9.7 & 37 & 44 & 12 & 0 & 7 \\
\hline 6 & 55 & 5.1 & 12.6 & 27 & 51 & 14 & 0 & 8 \\
\hline 7 & 42 & 6.1 & 61.8 & 41 & 45 & 5 & 2 & 7 \\
\hline 8 & 30 & 6.0 & 21.4 & 35 & 50 & 7 & 1 & 7 \\
\hline 9 & 43 & 5.0 & 19.4 & 66 & 24 & 4 & 3 & 3 \\
\hline 10 & 47 & 6.1 & 25.8 & 87 & 23 & 4 & 0 & 6 \\
\hline 11 & 46 & 5.2 & 24.2 & 54 & 35 & 8 & 0 & 2 \\
\hline 12 & 70 & 5.0 & 21.2 & 47 & 27 & 0 & 17 & 9 \\
\hline 13 & 68 & 5.2 & 21.1 & 44 & 43 & 5 & 0 & 0 \\
\hline 14 & 59 & 6.1 & 20.0 & 26 & 39 & 21 & 0 & 14 \\
\hline 15 & 60 & 5.1 & 27.8 & 55 & 37 & 2 & 0 & 6 \\
\hline 16 & 53 & 5.0 & 23.8 & 81 & 13 & 0 & 0 & 6 \\
\hline 17 & 50 & 10.0 & 23.4 & 45 & 49 & 4 & 0 & 2 \\
\hline 18 & 50 & 5.0 & 23.2 & 74 & 17 & 6 & 0 & 3 \\
\hline 19 & 46 & 5.0 & 11.6 & 56 & 22 & 12 & 1 & 9 \\
\hline 20 & 43 & 5.0 & 25.0 & 4 & 68 & 24 & 0 & 4 \\
\hline 21 & 46 & 5.2 & 31.0 & 27 & 53 & 12 & 0 & 8 \\
\hline 22 & 43 & 5.0 & 19.4 & 66 & 24 & 4 & 3 & 3 \\
\hline 23 & 30 & 5.5 & 20.8 & 41 & 10 & 41 & 4 & 4 \\
\hline MEDIA & $\mathbf{4 9 . 3 9}$ & $\mathbf{5 . 5 6}$ & $\mathbf{2 2 . 5 5}$ & $\mathbf{5 0 . 4 8}$ & $\mathbf{3 5 . 3 5}$ & $\mathbf{1 1 . 5 7}$ & $\mathbf{1 . 6 5}$ & $\mathbf{5 . 8 7}$ \\
\hline
\end{tabular}

Tabla 2.- Parámetros hematológicos de los guajolotes criollos de traspatio.

Variables

Hematocrito \%

Proteínas plasmáticas $\mathrm{g} / \mathrm{dl}$
Guajolotes $(\mathrm{X} \pm$ DS)

$49.39 \pm 11.13$

$5.56 \pm 1.12$
Valores de referencia

(Dein et al., 1982) $45.90-36.36$
$6.30-3.80$ 


\begin{tabular}{ccc}
\hline Leucocitos totales $\times 103 / \mu 1$ & $22.55 \pm 10.23$ & 20 \\
\hline Linfocitos \% & $50.48 \pm 22.46$ & $90-54$ \\
\hline Heterófilos \% & $35.35 \pm 18.63$ & $40-10$ \\
\hline Eosinófilos \% & $11.57 \pm 11.47$ & $4-0$ \\
\hline Basófilos \% & $1.65 \pm 3.60$ & $1-0$ \\
\hline Monocito \% & $5.87 \pm 3.17$ & $5-0$ \\
\hline
\end{tabular}
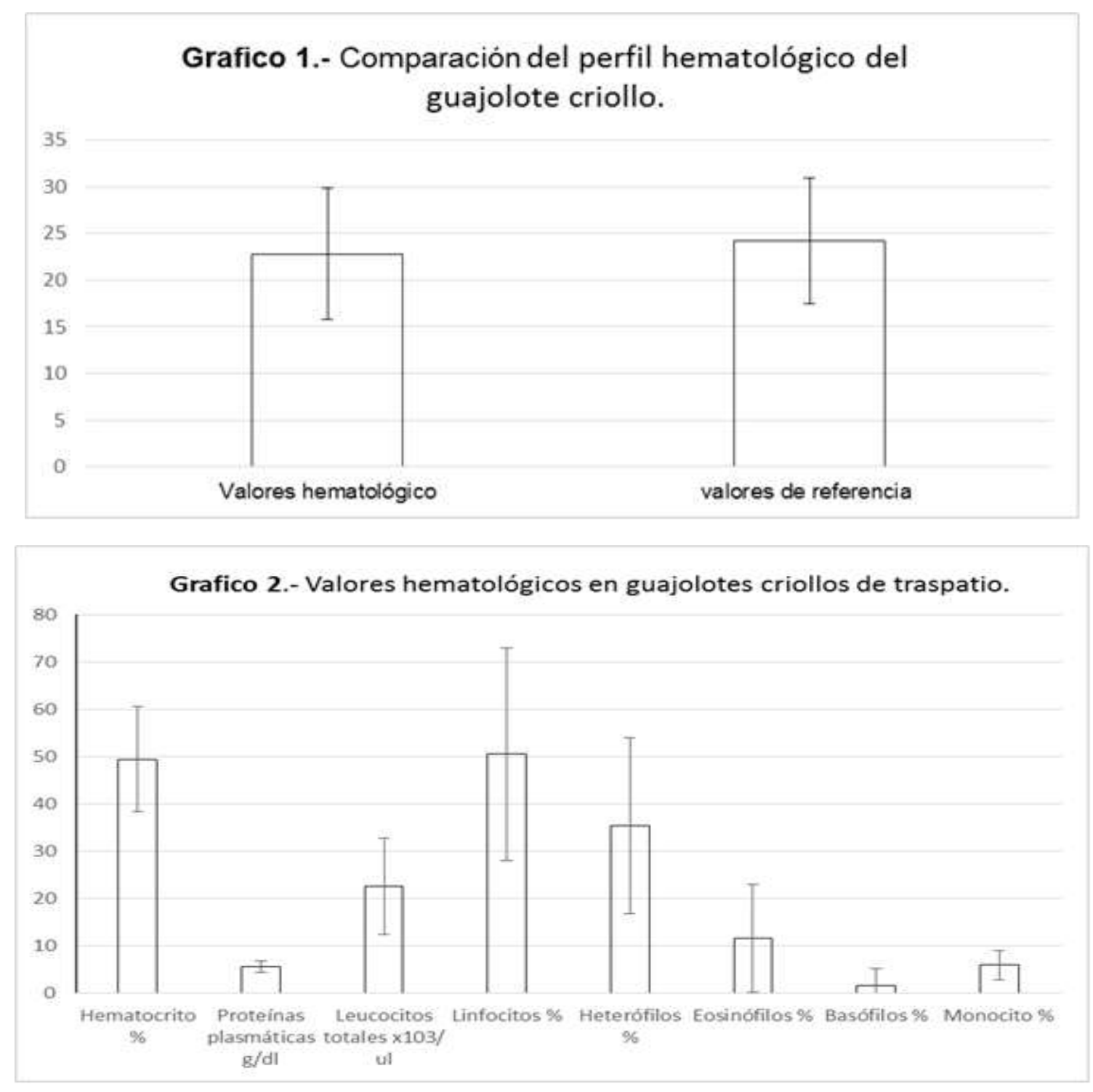

\section{CONCLUSIÓN}

El desarrollo de valores de referencia para hematología obtenidos a partir de los guajolotes criollos sanos de las unidades familiares de traspatio, utilizados en esta investigacion, son de gran importante debido a que estos proveen la línea base e información diagnóstica que permite evaluar el estado de salud o enfermedad de los animales. La presente información hematológica de los guajolotes criollos en las condiciones

Revista Científica Biológico Agropecuaria Tuxpan 5 (2) 
descritas es la base para futuras investigaciones sobre esta especie animal, en particular para los estudios de resistencia a enfermedades y otros factores ambientales.

\section{LITERATURA CITADA}

Borjesson, D. L., Christopher, M. M., \& Boyce, W. M. (2000). Biochemical and hematologic reference intervals for free-ranging desert bighorn sheep. Journal of Wildlife Diseases, 36(2), 294-300. https://doi.org/10.7589/0090-3558-36.2.294

Cetin, N., Güçlü, B. K., \& Cetin, E. (2005). The effects of probiotic and mannanoligosaccharide on some haematological and immunological parameters in turkeys. Transboundary and Emerging Diseases, 52(6), 263-267.

https://doi.org/10.1111/j.1439-0442.2005.0073

Dein, F. J. (1982). Avian clinical hematology. Proceedings of the Association of Avian Veterinarians. Emory University Press, Atlanta, Georgia, 5-29.

Drew, M. L. (2003). Galliformes (pheasants, grouse, quail, turkeys, chacalacas, currasows, hoatzins). Zoo and wild animal medicine, 5th ed., WB Saunders Co, UK P. 161-171.

Franzmann, A. W., \& Leresche, R. E. (1978). Alaskan moose blood studies with emphasis on condition evaluation. The Journal of Wildlife Management, 42(2), 334-351.

https://doi.org/10.2307/3800270

García-Montijano, M., García, A., Lemus, J. A., Montesinos, A., Canales, R., Luaces, I., \& Pereira, P. (2002). Blood chemistry, protein electrophoresis, and hematologic values of captive Spanish imperial eagles (Aquila adalberti). Journal of Zoo and Wildlife Medicine, 33(2), 112-117.

https://doi.org/10.1638/1042-7260(2002)033[01

Girish, C. K., Smith, T. K., Boermans, H. J. and Karrow, N. A. (2005c). Effects of Feeding Blends of Grains Naturally Contaminated With Fusarium Mycotoxins on Performance,
Reséndiz et al., 2017

Hematology, Metabolism, and Immunocompetence of Turkeys. Poultry Science, 87: 421-432.

Hafez, H. M., Hagen, N., \& Allam, T. S. (2016). Influence of stocking density on health condition in meat turkey flocks under field conditions. Pakistan Veterinary Journal, 36(2), 134-139.

Hochleithner, M. (1994). Biochemistries. Avian medicine: principles and application. Estados Unidos de Norteamerica, Wingers Publishing Inc. P. 223-245.

Lashev, L., Atanasova, S., \& DINEV, T. (2015). Interspecies and gender-related variations of some haematological parameters in galliformes bird species. Bulgarian Journal of Veterinary Medicine, 18(4), 325-337. https://doi.org/10.15547/bjvm.783

Lochmiller, R. L., \& Grant, W. E. (1984). Serum chemistry of the collared peccary (Tayassu tajacu). Journal of wildlife diseases, 20(2), 134140. https://doi.org/10.7589/0090-3558-20.2.

López, C. C. (1997). Determinación de los parámetros hematológicos en pollos de engorda criados a diferentes altitudes: susceptibilidad al síndrome ascítico de diferentes estirpes genéticas de pollos de engorda. Tesis doctorado. Facultad de Medicina Veterinaria y Zootecnia. U.N.A.M. P.

Maxwell, M. H. (1993). Avian blood leucocyte responses to stress. World's Poultry Science Journal, 49(1), 34-43.

https://doi.org/10.1079/WPS19930004

Nietfeld, M. T. (1994). Wildlife marking techniques. Research and management techniques for wildlife and habitats. Estados Unidos, The Wildlife Society. P. 140-168.

Ognik, K., Sembratowicz, I., \& Czech, A. (2015). The effect of aloe preparation and 5-oxo-1, 2, 4triazine on immunological and haematological 
indices of blood of turkey hens subjected to stress. Acta Veterinaria Brno, 84(4), 365-371. https://doi.org/10.2754/avb201584040365

Peinado, V. I., Polo, F. J., Celdrán, J. F., Viscor, G., \& Palomeque, J. (1992). Hematology and plasma chemistry in endangered pigeons. Journal of Zoo and Wildlife Medicine. 23(1):65-71.

Rosskopf, W. Jr.; Woerpel, R. (1991). Pet avian hematology trends. Proceedings of the annual Conference. Asociation of Avian Veterinarians. 98-111.
Tell, L. A., \& Citino, S. B. (1992). Hematologic and serum chemistry reference intervals for Cuban Amazon parrots (Amazona leucocephala leucocephala). Journal of Zoo and Wildlife Medicine, 62-64.

West, G. D., \& Haines, V. L. (2002). Hematology and serum biochemistry values of captive Attwater's prairie chickens (Tympanuchus cupido attwateri). Journal of zoo and wildlife Medicine, 33(2), 122-124. https://doi.org/10.1638/1042-7260(2002)033[01
Copyright (c) 2017 Roberto Reséndiz Martinez, Salvador Romero Castañón,

Herm inio I. Jiménez Cortez y Briseida Lucia Castro Bautista

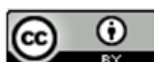

Este tex to está protegido por una licencia licencia Creative Commons $\underline{4.0}$

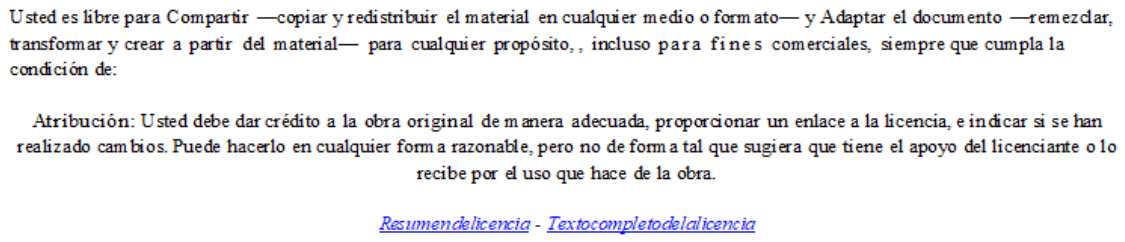

Usted es libre para Compartir —copiar y redistribuir el material en cualquier medio o form ato-y Adaptar el documento $\rightarrow$ remezclar, transformar y crear a partir del material- para cualquier propósito,, incluso para fines comerciales, siempre que cumpla la

Atribución: Usted debe dar crédito a la obra original de manera adecuada proporcionar un enlace a $\underline{\text { Resumendelicencia - Textocompletocielalicencia }}$ 